\title{
6th International Consensus Conference on the Treatment of Primary Breast Cancer
}

\author{
February 25-28, 1998, St. Gallen, Switzerland
}

\section{Introduction}

Breast cancer is a heterogeneous disease. The choice of today's primary treatments is based on criteria of the patient, the tumor, and the response to treatment. We have learned that the medical and social environment of patients contribute to this heterogeneity with respect to the interpretation of the available data and cost benefit of treatments. All of our knowledge about the selection of therapies is derived from results of case series and randomized clinical trials. Since the 5th International Conference on Adjuvant Therapy of Primary Breast Cancer 1995 in St. Gallen, Switzerland, serveral new concepts and treatment strategies have been investigated. Some of these can help the treating physician better selecting a treatment for an individual patient with breast cancer. In this summary we describe some highlights which were presented and discussed at the 6th International Conference on Adjuvant Therapy of Primary Breast Cancer, held in St. Gallen, Switzerland, in February 1998. Special attention is given to areas of ongoing research and update on treatment recommendation.

The following questions were asked and discussed by the panel participants:

\section{Question 1: Which Prognostic Factors Should be Used in Node-Negative and Node-Positive Disease?}

Pathological tumor size $>$ grade $>$ estrogen receptor $(E R)$ and progesteron receptor $(\mathrm{PgR})$ status and age have been identified as the most important prognostic factors in node-negative disease. Obviously the nodal status is important but correlates usually with tumor size and applies only to node-positive disease. Adequate nodal status can only be determined if at least 10 axillary lymph nodes are excised and examined. Patients with age above 35 years and negative nodal status as defined above and tumors smaller than $1 \mathrm{~cm}$ with favorable grade and positive ER and/or PgR status usually do not need further therapy. There was also a question whether the age limit for this recommendation should be 40 years instead of 35 years. S-phase determination, e.g. assessed by flow cytometry, was also addressed by the panel. There was an agreement that there is no role for S-phase determination in the routine management of breast cancer patients.

ER cutoff limits in immunohistochemistry were also discussed. No clear agreement could be reached. Investigations of two research groups support a cutoff of $\geq 10 \%$ positive cells, irrespective of the intensity of staining. Scandinavian data support the finding that $10 \%$ cutoff for positivity is comparable with the $10 \mathrm{fmol} / \mathrm{mg}$ cut off using biochemical analysis. Other panel discussions from the USA supported the use of institutional cutoff values to identify hormone-insensitive disease.

Further prognostic factors were considered. PgR and vessel invasion were discussed. It was concluded that $\mathrm{PgR}$ should also be taken in consideration in order to determine whether the disease of a patient is potentially endocrine sensitive.

\section{Question 2: Predictive Factors to Respond to Adjuvant Therapy?}

There was a consensus that except for $\mathrm{ER} / \mathrm{PgR}$ we presently do not have such a tool. c-erb2 which was reported to indicate tamoxifen and CMF chemotherapy resistance has so far only been tested retrospectively.

Furthermore, there is presently no predictive factor to response to preoperative chemotherapy.

The response of the primary tumor, especially pathological complete remission, to preoperative treatment was found to be important but still investigestional. Furthermore, the response to primary nonsurgical treatment could rather be a prognostic factor, not a predictive factor.

\section{Question 3: Sentinel Axillary Biopsy}

This question needed a substantial time of the discussion. Different opinions between the Western and Eastern side of the Atlantic emerged during the discussion. European surgeons stated that this procedure is clearly investigational. American surgeons felt that sentinel biospy will slowly but surely invade the field of axilla surgery irrespective of whether long-term safety data are available or not. However, sentinel biopsy 
should only be performed in experienced hands as defined as $\geq 50$ negative cases without 1 failure. Furthermore, it was agreed that stopping axillary surgery in case of positive sentinel biopsy is investigational. Several European experts insisted on the removal of $\geq 10$ nodes as an adequate axillary surgery because the adequate estimation of the risk of recurrence depends rather on the proportion of involved nodes as compared to absolute numbers. These experts supported their opinion by the fact that patients with 5 excised nodes have an inferior long-term prognosis than patients with 10 or more excised nodes. There seems to be a close correlation between the number of excised/examined nodes and the risk of long-term recurrence, at least in the range between 0 and 8 nodes.

\section{Question 4: Who Should have Neoadjuvant Chemotherapy in Operable Breast Cancer?}

During the discussion of this question again a gap between American surgeons and European experts emerged. Martine Piccart expressed that operable breast cancer should be operated primarily, and Michael Baum added that primary surgery of operable breast cancer usually results in a pathological complete remission rate of nearly $100 \%$. Most panel participants felt that primary chemotherapy is still investigational. American surgeons answered that the use of neoadjuvant chemotherapy should depend on the tumor size/breast size ratio. Their recommendation was to consider primary chemotherapy in all patients in which mastectomy is planned. A word of caution was also addressed because of the unknown influence of primary systemic antineoplastic treatment on known valid prognostic factors for postoperative adjuvant therapy.

Primary medical treatment might also be endocrine therapy. Preliminary results are encouraging.

\section{Question 5: Radiotherapy after Mastectomy}

J. Kurtz stated that postoperative radiotherapy gives a 4-fold reduction of locoregional failure. Adjuvant radiotherapy after mastectomy was felt to be indicated in cases with a risk of local relapse of around $40 \%$ as observed in a recently published large trial.

In cases with a locoregional risk of failure of around $20 \%$ or higher radiotherapy after mastectomy should be considered but it can not be unanimously recommended on the basis of presently available data. New trials addressing the question are needed in this patient population (e.g. $>4$ nodes).

Radiotherapy to internal mammary nodes is investigational. It should not be used as a routine outside clinical trials until results of the ongoing EORTC trial addressing this question are available.

There was a consensus regarding the statement that no radiotherapy should be used in node-negative disease after mastectomy.

To summarize: One cannot conclude for all patients what has been proved for a very selected patient population using partially an inadequate surgical procedure and a systemic adjuvant therapy which is inadequate to what is presently known. It is recommended to look at the evidence of all trials of radiotherapy (overview 1995) and to consider also the effect of adequate systemic treatment. The use of radiation therapy in patients receiving anthracycline- and/or taxane-containing chemotherapy is investigational in terms of benefit and safety.

\section{Question 6: Who Should not Receive Adjuvant Therapy in Node-Negative Disease?}

Usually patients with tumors of less than $1 \mathrm{~cm}$ pathological size and grade 1 are considered. However, it was stated that most women accept an adjuvant chemotherapy of CMF $6 \times$ for a gain of $1 \%$ in overall survival at 10 years (at least in Australia). Patients should be asked for their preference. The conclusion was that this minimal risk $(<10 \%$ treatment failure at 10 years) should be discussed with the patients, especially with patients of younger age. This 'minimal' risk will be decreased by the use of adjuvant systemic treatment. Obviously the absolute gain is small but real. See also question 7 .

\section{Question 7: Good Risk/High Risk in Node-Negative Disease}

As expected there was a lively discussion. National Surgical Adjuvant Project for Breast and Bowel Cancers (NSABP) results showed that all patients will benefit from chemotherapy. No subgroup who did not benefit could be identified but in tumors $<1 \mathrm{~cm}$ the effect of chemotherapy is unknown. However, small gains might be important even if not shown in multivariate analysis of a large single trial. There is a lot of heterogeneity in tumors between 1 and $2 \mathrm{~cm}$. The present evidence should be discussed with patients, and patients' preference should be taken into account. However, it also was very clear that patients' preference is heavily dependent on physicians bias. New methods are developed to inform the patients. These new tools might be helpful in minimizing the influence of physicians bias on the patients' preference.

The present evidence was summarized as follows: If anthracycline-containing regimens are added to tamoxifen, chemoendocrine therapy is usually superior to endocrine treatment. If CMF is added, only original CMF regimens using day 1 and day 8 drug administration proved to be superior to tamoxifen alone. None of the other trials using CMF modifications showed superiority of chemoendocrine treatment as compared to endocrine treatment alone. The overview showed clearly a benefit for chemotherapy in this risk group, but the overview probably underestimates the magnitude of the benefit due to the 'dilution' of results generated by studies using 'inadequate' chemotherapy. On the other hand, it was felt that chemoendocrine treatment or chemotherapy to all of these patients with intermediate risk is probably not an appropriate recommendation. Again patients' preference should be taken in consideration. In the high-risk group chemotherapy and tamoxifen is the standard. 


\section{Question 8: High-Risk Node-Negative, ER-Negative disease}

All patients should receive chemotherapy. In PgR-positive disease patients should receive tamoxifen. In ER- and PgRnegative disease no tamoxifen treatment is indicated.

\section{Question 9: Premenopausal, Node-Positive Disease}

American investigators felt that chemotherapy is still standard, and in ER- and/or PgR-positive disease tamoxifen should be added to the treatment. Some European experts agreed but other experts felt that this might not be a standard because of unknown long-term side effects, even if the combined chemoendocrine treatment is more efficacious. American investigators did not agree and expressed their opinion that the situation is unclear. There was an agreement that the induction of amenorrhea is of value regarding efficacy.

Question 10: Endocrine Treatment Alone in Node-Positive, Premenopausal Patients?

Ovarian Ablation Alone

North American investigators stated that ovarian ablation alone is an adequate treatment in (high) ER-positive disease and might be even superior to chemotherapy alone. It is also the only treatment available in many places throughout the world. It was discussed that tamoxifen would be just as efficacious, but this recommendation would be based on indirect comparisons. So far, ovarian ablation is the standard until we have results available from randomized trials comparing ovarian ablation with tamoxifen. Again it was stated that the benefit of ovarian ablation is probably underestimated.

\section{Tamoxifen Alone}

European experts, mainly of more Northern origin, agreed that tamoxifen alone could be an option, especially for patients $>40$ years. However, most other experts felt that chemoendocrine treatment should be given due to the fact that the combination is better in almost all published trials. It was added that the same is true for ovarian ablation.

\section{Question 11: Postmenopausal, Node-Positive, ER-Positive Disease}

Again NSABP data supported the use of chemotherapy in this subgroup, and it was stated that anthracyclines should be used when chemotherapy is given. On the other hand, it was also mentioned that the overview showed an age-related effect of benefit. European experts claimed that this subgroup is also very heterogeneous, and we actually do not have the real answer at present.

Regarding concomittant or sequential use of chemoendocrine treatment, it was agreed that this question cannot be answered definitely until we have the results of the ongoing Intergroup trial. Chemoendocrine treatment in ER/PgR-negative disease was found to be not adequately studied but so far has no place in the adjuvant treatment of breast cancer. However, further research is still needed, also addressing the question of the prevention of contralateral breast cancer.

\section{Question 12: Standard in Adjuvant Chemotherapies}

CMF chemotherapy is still an adequat treatment also in node-positive disease. Adequate treatments are CMF $6 \times$ and AC $4 \times$.

Anthracycline-containing regimens showed good results, especially in the postmenopausal patient population. However, present results of 2 trials comparing CMF type regimens with 'negative' results and 1 'positive' trial using nonequitoxic treatments are available. It was also stated that recommendations to use anthracycline-containing regimens (e.g. CAF instead of $\mathrm{CMF}$ ) would therefore base on indirect comparisons.

There is no role for using taxanes in the adjuvant treatment. There is no role for dose-dense regimens or treatments with higher dose intensity. There was no recommendation regarding the sequence with radiotherapy.

\section{Conclusion}

These recommendations are guidelines and not definitive statements. They reflect a personal view of the author of a consensus based on currently available scientific information. The prognosis and the estimation of the treatment impact and of the burden of the proposed treatment should be discussed with each individual patient. Whenever possible, patients should be treated in clinical trials with adequate sample size in order to enlarge our knowledge and to improve therapy of primary breast cancer.

B. Thürlimann, St. Gallen 